\title{
Wireless Sensing in Complex Electromagnetic Media: Construction Materials and Structural Monitoring
}

\author{
Burak Ozbey, Student Member, IEEE, Hilmi Volkan Demir, Senior Member, IEEE, Ozgur Kurc, \\ Vakur B. Ertürk, Member, IEEE, and Ayhan Altintas, Senior Member, IEEE
}

\begin{abstract}
In this paper, wireless sensing in the presence of complex electromagnetic media created by combinations of reinforcing bars and concrete is investigated. The wireless displacement sensing system, primarily designed for use in structural health monitoring (SHM), is composed of a comb-like nested split-ring resonator (NSRR) probe and a transceiver antenna. Although each complex medium scenario is predicted to have a detrimental effect on sensing in principle, it is demonstrated that the proposed sensor geometry is able to operate fairly well in all scenarios except one. In these scenarios that mimic real-life SHM, it is shown that this sensor exhibits a high displacement resolution of $1 \mu \mathrm{m}$, a good sensitivity of $7 \mathrm{MHz} / \mathrm{mm}$ in average, and a high dynamic range extending over $20 \mathrm{~mm}$. For the most disruptive scenario of placing concrete immediately behind NSRR, a solution based on employing a separator behind the probe is proposed to overcome the handicaps introduced by the medium. In order to obtain a one-to-one mapping from the measured frequency shift to the displacement, a numerical fit is proposed and used. The effects of several complex medium scenarios on this fit are discussed. These results indicate that the proposed sensing scheme works well in real-life SHM applications.
\end{abstract}

Index Terms-Wireless passive sensor, displacement sensor, strain sensor, nested split ring resonator (NSRR), concrete,

Manuscript received January 13, 2015; revised May 20, 2015; accepted May 22, 2015. Date of publication June 4, 2015; date of current version August 12, 2015. This work was supported by the Scientific and Technological Research Council of Turkey (TÜBITAK) through Elektrik Elektronik ve Enformatik Araştırma Grubu (EEEAG) under Grant no: 112E255. The work of H. V. Demir was supported in part by the European Science Foundation through the European Young Investigator Awards, and in part by the Turkish National Academy of Sciences. The associate editor coordinating the review of this paper and approving it for publication was Dr. Stefan J. Rupitsch.

B. Ozbey is with the Department of Electrical and Electronics Engineering, Bilkent University, Ankara 06800, Turkey (e-mail: ozbey@ee.bilkent.edu.tr).

H. V. Demir is with the Department of Electrical and Electronics Engineering and the Department of Physics, Institute of Materials Science and Nanotechnology, Bilkent University, Ankara 06800, Turkey, and also with the School of Electrical and Electronic Engineering and the School of Physical and Mathematical Sciences, Nanyang Technological University, Singapore 639798 (e-mail: volkan@stanfordalumni.org).

O. Kurc is with the Department of Civil Engineering, Middle East Technical University, Ankara 06800, Turkey (e-mail: kurc@metu.edu.tr).

V. B. Ertürk is with the Department of Electrical and Electronics Engineering, Bilkent University, Ankara 06800, Turkey (e-mail: vakur@ee.bilkent.edu.tr).

A. Altintas is with the Communications and Spectrum Management Research Center, Department of Electrical and Electronics Engineering, Bilkent University, Ankara 06800, Turkey, and also with the Faculty of Engineering and Natural Sciences, Abdullah Gul University, Kayseri 38039, Turkey (e-mail: altintas@ee.bilkent.edu.tr).

Color versions of one or more of the figures in this paper are available online at http://ieeexplore.iee.org.

Digital Object Identifier 10.1109/JSEN.2015.2441555 reinforcing bar (rebar), complex medium, structural health monitoring (SHM).

\section{INTRODUCTION}

$\mathbf{E}$ NABLING telemetric measurements, wireless passive sensors are increasingly desired in numerous applications including measurements of stress [1], [2], strain [3]-[5], displacement [5], [6], temperature [7], [8], humidity [9], [10] and $\mathrm{pH}$ [8], [11], [12], and detection of chemical compounds [13], [14]. Especially, in the area of structural health monitoring (SHM), it can be a very cumbersome task to use different cables for each strain gage at data acquisition systems employed to monitor buildings or other structures. Additionally, tearing down part of a wall or a construction to reach the sensing structure for battery replacement is both difficult and costly. Hence, the sensor preferably needs to be wireless and passive in order to be conveniently embedded inside walls and cast into concrete [15].

In SHM, the peak strain rising from displacement is one of the prime damage indicators [16]. Hence, keeping track of strain forming on such structures provides valuable information about the level of overall deformation and possibly whether the evacuation of a building will or will not be necessary. Recently, wireless passive strain and displacement sensors have been shown, some of which were specifically designed for remote SHM [17]-[25]. In [17], the authors benefited from a patch antenna as a sensing structure, and by using another antenna as an interrogator, they wirelessly performed strain sensing and crack detection. The underlying principle is that the antenna, subject to a change with a crack or strain forming on it, changes its resonant frequency due to its dimensions comparable to the crack or strain induced deformation. In [18] and [24], an RFID reader and a tag were used for interrogation. In [18], the tag was in the form of a meander-line antenna; and in [24], a two-part structure with an open-circuit microstrip loaded by sensing notches and a selector that can move with the applied force was used as the tag. In both reports, the resolution was experimentally shown to be around sub-mm level, while for the latter one it was found that resolution levels as low as a few tens of $\mu$ m's were theoretically possible with certain RFID readers. In [19], a circular microstrip patch antenna was employed as the sensing structure, but a maximum interrogation distance of $5 \mathrm{~cm}$ was 


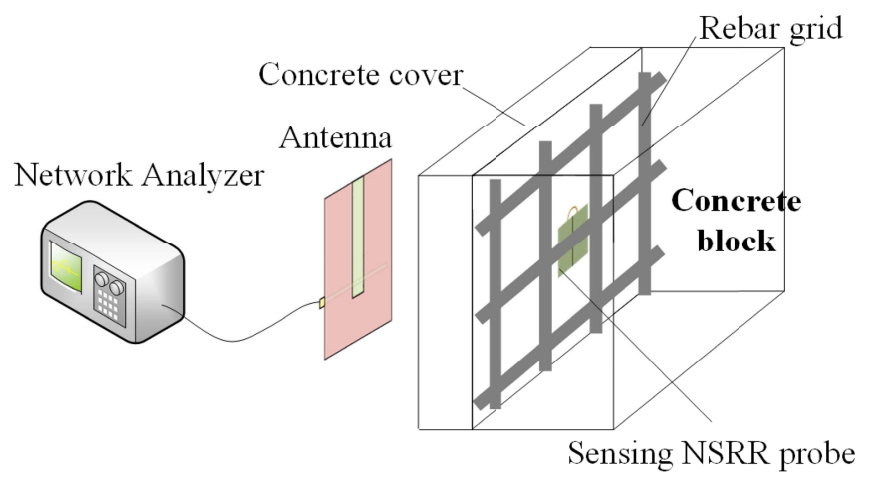

Fig. 1. Schematics of the sensor geometry used in this work.

experimentally shown. In [20], the authors demonstrated a wireless strain transducer based on a microstrip patch antenna that is loaded with two open loops to sense the strain in two directions. The sensitivity of the proposed structure, which is defined as the measured frequency shift per unit strain induced, was shown to be $2.35 \%$ change in frequency for $1.00 \%$ strain.

In real-life applications, however, the complex medium constituted by combinations of concrete and rebar grid generally has a detrimental effect on RF wireless sensors that interrogate backscattered fields. In order for a sensor to qualify for use in a real-life application, first the question of whether it can function well in the presence of such a medium should be addressed. Among the wireless passive strain and displacement sensors previously reported, this effect has not been systematically considered. Although the electromagnetic properties (relative dielectric constant, loss factor, loss tangent, conductivity, etc.) as well as the transmission and reflection properties of rebar grid, concrete and reinforced concrete in RF and microwave frequencies have been studied extensively in literature [26]-[32], wireless sensing in their medium has not been demonstrated. It was shown by Dalke et al. that transmission through or reflection from a rebar grid is closely related with the unit cell length or period of the grid [28]. It was also demonstrated that above a certain free space wavelength approximately equal to this period, a metal rebar grid completely reflects the incident wave, acting as a perfect electric conductor [28]. Dalke et al. also derived that a conducting concrete block produces periodic transmission maxima when the guided wavelength inside the concrete $\left(\lambda_{\mathrm{c}}\right)$ is equal to $2 W, W, W / 2, W / 4, \ldots$, where $W$ is the thickness of the concrete. In the case of periodically reinforced concrete, these two elements are combined and their characteristics are superimposed. The resulting behavior is a more complicated function of both $W$ and the rebar grid period [28].

Recently, a novel wireless and passive displacement and strain sensor configuration for SHM has been proposed by our group [33], [34]. This sensor basically works by the principle of creating a coupled system between an NSRR probe and an antenna. The sensing probe structure is attached to the object on which the axial displacement between two points are to be measured (see Fig. 1). The antenna serves both as a receiver and a transmitter, sending the signal driven by the network analyzer. Previously, the sensor was shown as a proof-of concept under laboratory conditions, i.e., working on a translation stage when the backside of the probe was loaded with free space [33]. The resolution of the sensor was shown to be sub- $\mu \mathrm{m}$, with a tracked displacement range up to $20 \mathrm{~mm}$, possessing a sensitivity level of $10 \mathrm{MHz} / \mathrm{mm}$ for a $2 \mathrm{~mm}$ range. In [34], the sensing probe was attached on a standard construction reinforcing bar, employed in critical regions of a building such as columns and beams. The rebar was pulled in a high-scale loading setup, where a force up to $36,7 \mathrm{kN}$ was applied. It was shown that the sensor could accurately detect the entirety of the elastic and plastic deformation regions of the rebar, with both a high dynamic range (up to $10 \%$ strain and $2 \mathrm{~mm}$ displacement) and a microstrain-level resolution in the elastic range. The experiments were also successfully repeated in the presence of a thin concrete cover between the antenna and the sensing probe.

In this paper, different from the works of our groups and others, the proposed sensor geometry is systematically studied and tested in a fully real-life compatible environment, where the sensing NSRR probe is loaded with several construction elements including a rebar grid, concrete and combinations of these elements, which constitute an electromagnetically complex medium. The effects of rebar and concrete on the sensing are understood first separately and then together. For such a sensing system, the method used to extract the displacement or strain information from the monitored frequency shift is also essential since this serves as a calibration for other measurements. Here, a numerical fit is introduced for this calibration, and the modification of the fit parameters due to the effects of the complex medium are shown experimentally.

The organization of the paper is as follows: In Section II, the sensor configuration is described and the working principles are briefly discussed. In Section III, the translation frequency shift characterization of the sensor in free space is given and a numerical fit to these measurements is provided. In Section IV, experiment results in the presence of rebar and concrete are shown and their effects on the fit parameters are discussed. Additionally, a solution to the problems created by the complex media is suggested. Section V concludes the paper.

\section{SENSOR GEOMETRY}

The proposed sensing system consists of two parts: A sensing probe designed in comb-like NSRR geometry and a transceiver antenna, which serves as the interrogator. The comb-like NSRR geometry, first proposed in [35], is selected primarily because of its higher localization of fields and its ease of design. Detailed information about the specific comb-like NSRR design used in the sensor can be found in [33] and [34]. The structure and the dimensions of the split comb-like NSRR geometry employed in this paper as well as in [33] and [34] are shown in Fig. 2. This geometry consists of a comb-like NSRR split symmetrically into two halves, which are electrically shorted by a thin jumper wire on the normally continuous uppermost tooth pair. Splitting the NSRR into two halves saves the system from strain propagation on NSRR, which is fabricated from a dielectric material (e.g., Rogers Duroid) and allows for larger displacements to be monitored. Strain information can also be retrieved from the measured 


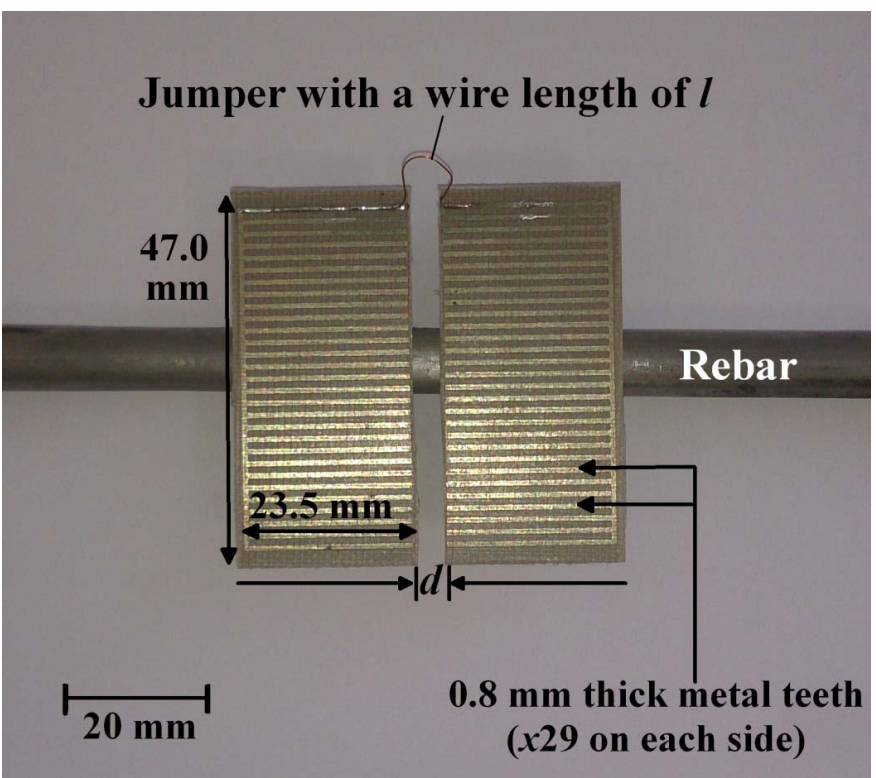

Fig. 2. Structure and dimensions of the split comb-like NSRR design used in this paper, shown attached on a rebar.

displacement since the relationship between displacement and strain is given by:

$$
e=\frac{\Delta D}{D}
$$

where $e$ is the engineering strain, $D$ is the distance between the two attachment points of the NSRR probe and $\Delta D$ is the change in $D$, which can be positive (in the case of an elongation) or negative (in the case of a contraction). The operation frequency is selected as $400 \mathrm{MHz}$. The relative permittivity of concrete is around 6 , and at this frequency the guided wavelength $\left(\lambda_{c}\right)$ is around $30 \mathrm{~cm}$. This $\lambda_{c}$ is found to be appropriate for RF communication since the concrete cover to be placed between the antenna and NSRR probe has a high transmission coefficient [28]. Moreover, the concrete reinforced with the rebar grid on which the probe is attached has a higher reflection coefficient at $400 \mathrm{MHz}$ [28]. Another advantage of the comb-like NSRR geometry is that the dimensions of the structure $(4.7 \mathrm{~cm} \times 4.7 \mathrm{~cm}$ footprint $)$ are much smaller with respect to the free space wavelength $(75 \mathrm{~cm})$. The antenna is based on the bandwidth-enhanced and size-reduced microstip monopole single-slot antenna design shown in [36]. The dimensions of the antenna are selected such that the antenna operates around $420 \mathrm{MHz}$ center frequency. The antenna is horizontally polarized, producing an E-field along the parallel teeth of the NSRR probe structure, which in turn increases the coupling. The antenna and the sensor are shown on the translation stage in Fig. 3.

In the proposed sensing system, the coupling between the NSRR probe and the antenna is achieved by placing the probe within the near-field of the antenna, and the displacement causes a local frequency peak or dip in the input impedance of this coupled system to shift. Because there is a one-to-one relationship between the displacement and the coupled system frequency, every frequency corresponds

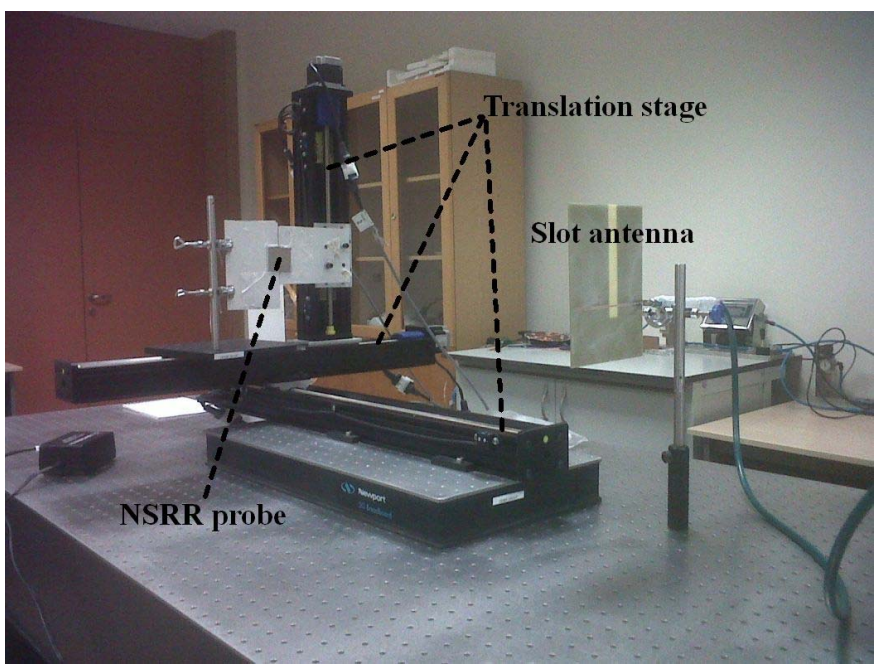

Fig. 3. The experiment setup of the sensing system, showing the NSRR probe on the translation stage and the microstrip slot antenna.

to a specific displacement. The near-field interaction between the antenna and the sensor results in an increased sensitivity (up to $10 \mathrm{MHz} / \mathrm{mm}$ ) and a higher resolution (sub- $\mu \mathrm{m}$ ), as shown in [33]. The system sensitivity and resolution naturally decrease as the monitoring distance $\left(D_{m}\right)$, which is the distance between the antenna and the NSRR probe, is increased. At a high $\mathrm{D}_{\mathrm{m}}$, increasing the input power is observed to be ineffective in recovering the sensitivity or resolution levels attained at low $\mathrm{D}_{\mathrm{m}}$ 's. The maximum monitoring distance $\left(\mathrm{D}_{\mathrm{m}, \text { max }}\right)$ is therefore around $60 \mathrm{~cm}$ in the experiments, which is below $\lambda_{0}(75 \mathrm{~cm}$ at $400 \mathrm{MHz}$ operating frequency) to ensure that the NSRR probe is in the near-field of the antenna.

\section{Measurements in Free Space}

There are two independent and controllable variables that determine the resonance frequency of the modified comb-like NSRR structure: 1) The end-to-end separation between the two electrically connected NSRR parts $(d)$, and 2$)$ the length of the jumper used to short the uppermost tooth (l) (see Fig. 2). $C_{0}$ and $L_{0}$ are the effective capacitance and effective inductance of the unmodified comb-like NSRR (which is not divided into two moving parts with a jumper wire connecting them but instead is rigid and has a continuous metal line absent of the jumper). $d$ and $l$ determine the capacitance and inductance, respectively, which in turn set a new $f_{0}$ for the whole structure, where $f_{0}$ is the effective resonance frequency of comb-like NSRR without a jumper, i.e., when $l=0$ and $d=0$. As expected, an increase in $d$ decreases the capacitance and thus increases the resonance frequency. Also, an increase in $l$ increases the inductance and decreases the resonance frequency. The measured change of frequency with displacement for a range of $0-10 \mathrm{~mm}$ (except a $5 \mathrm{~mm}$ range for the sensor whose $l$ is $1 \mathrm{~cm}$ ), which is obtained on the translation stage with no concrete or rebar included in the scenario, is shown for different jumper lengths in Fig. 4.

It can be observed in Fig. 4 that increasing the jumper length is effective in decreasing the resonance frequency for 


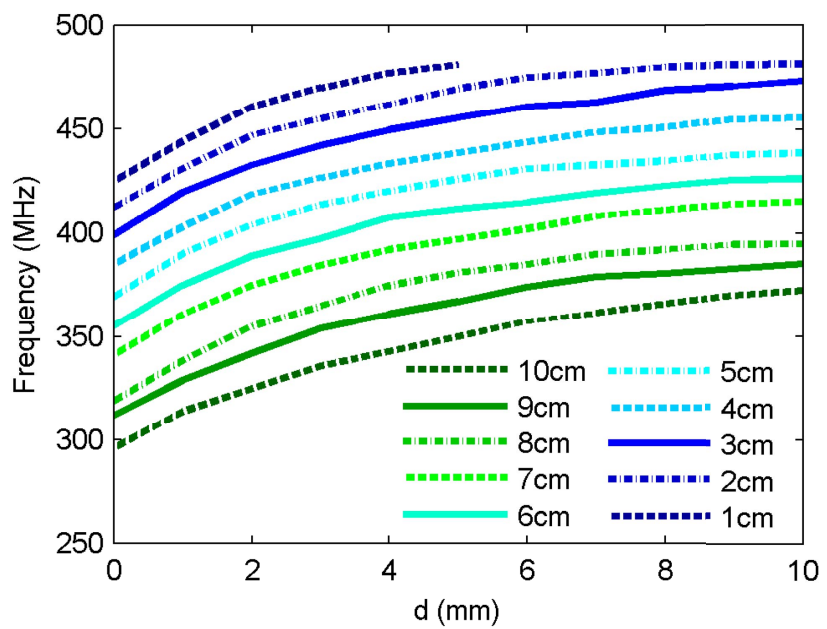

Fig. 4. Measured sensor resonance frequency as a function of displacement $(d)$, shown for different jumper lengths $(l)$. Measurement performed on a translation stage, where no rebar or concrete is present around the sensor elements.

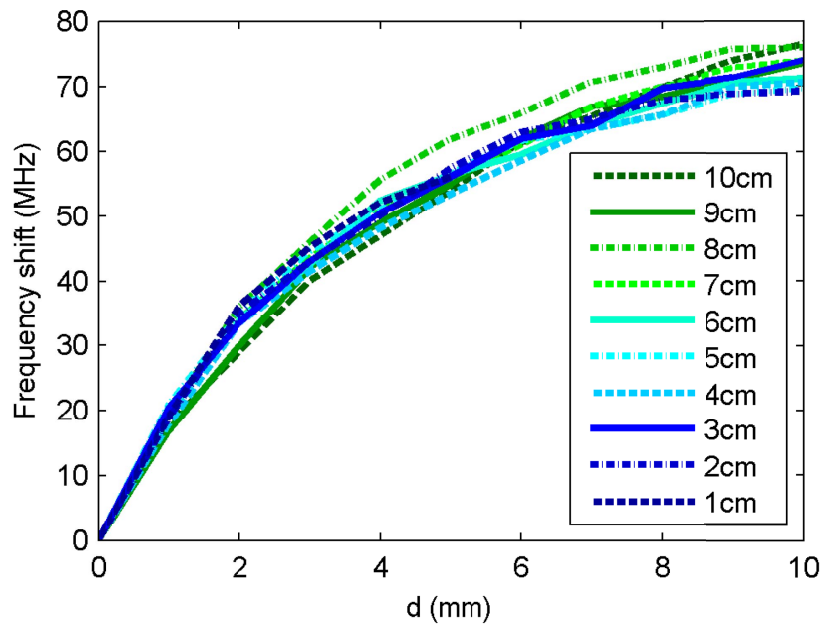

Fig. 5. Measured change of sensor resonance frequency with displacement $(d)$ for different jumper lengths $(l)$, where $f(d=0)$ is subtracted from each curve. Measurement performed on a translation stage, where no rebar or concrete is present around the sensor elements.

any $d$ value more or less by the same ratio. This becomes clear when all curves are plotted on top of each other by subtracting from each curve their corresponding $f(d=0)$ values as shown in Fig. 5. This figure also shows that the change of frequency with $d$ does not depend on $l$, meaning that the capacitance is independent of $l$ and is only altered by $d$.

As mentioned before, the calibration method is essential to extracting the displacement and strain information from the measured frequency shift. Although the relationship between the frequency and $d$ was shown to be highly linear $\left(R^{2}>0.99\right.$ over $\left.5 \mathrm{~mm}\right)$ for specific regions in the displacement range in [33]; for the entire range that can be detected by the sensor, it resembles a $\left(1-e^{-x}\right)$ type of curve as can be observed in Fig. 4. This is valid for any of the scenarios shown in [33] and [34]. For the purpose of calibration, a numerical fit can thus be applied to these curves as follows:

$$
f_{f i t}=\frac{k_{1}}{B}\left(1-e^{-B d}\right)+k_{2}
$$

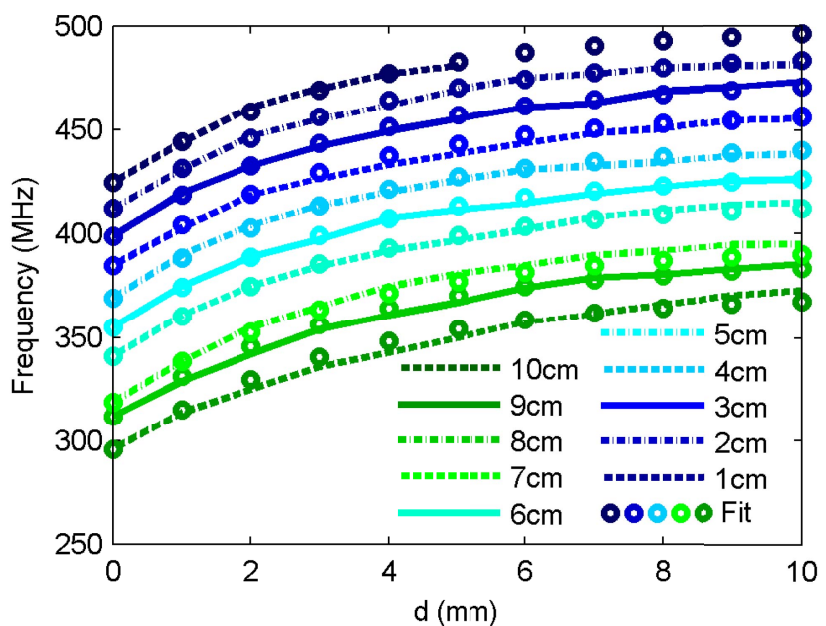

Fig. 6. Measured and fitted change of sensor resonance frequency for varying $d$, parametrized with respect to $l$. The numerical fit is exponential, which is plotted for each $l$. Measurement performed on a translation stage, where no rebar or concrete is present around the sensor elements.

In 2, the parameters $k_{1}$ and $B$ are constants, and remain the same for each $l$ value and for the whole $d$ range. $k_{2}$, however, is the offset frequency value which is experimentally shown to be linearly related to $l$ and to $f_{0}$ as:

$$
k_{2}=f_{0}-m l
$$

where $m$ is the slope of the $k_{2}$ versus $l$ linear relation shown in 3. Note that, it is thus experimentally revealed that the relationship between $f$ and $l$ is also linear. For the translation stage measurements in free space, the values that yield the most convenient fit are found as $k_{1}=22.5 \mathrm{MHz} / \mathrm{mm}$, $B=0.3 \mathrm{~mm}^{-1}$, and $m=14 \mathrm{MHz} / \mathrm{cm}$, for a measured $f_{0}$ of $437 \mathrm{MHz}$. The fits with these values are plotted for each $l$ in Fig. 6.

It has been experimentally demonstrated by Fig. 5 that the capacitance is independent of $l$ and only depends on $d$. It is also clear that the inductance is independent of $d$ and only depends on $l$. Therefore, $d$ and $l$ are two independent design parameters that set the resonance frequency by changing the overall $C$ and $L$, respectively. On the other hand, the presence of a complex electromagnetic medium is also effective in changing the overall $C$, which results in an altered resonance frequency. In the next section, measured impacts of several combinations of the rebar grid and concrete block on the sensor are shown, and their effects on the fit parameters are discussed.

\section{Measurements in Complex Media}

In the previous section, the measurement medium was free space and no element that could disrupt the sensor performance like a rebar grid or a concrete wall was present in the vicinity of neither the antenna nor the NSRR probe. In a real-life scenario, such a sensing structure should either be embedded in concrete or attached to the rebar just at the edge of the concrete wall. The NSRR probe is designed for use in the latter case, where its backside, which does not contain a 
ground plane or metal strips, is fastened to the rebar. Since the rebar is just at the edge of the concrete, its backside also makes contact with concrete, as shown in Fig. 1. In addition, as a typical real-life application, there exists a relatively thin concrete cover between the antenna and the NSRR probe. This in turn leaves the NSRR probe completely confined within concrete. The cover and the reinforced concrete constitute a complex electromagnetic medium, and this medium affects the characteristics of the sensor, including the shifting regime of frequency with displacement. In this section, results of various experiments are presented for several scenarios that involve combinations of these elements.

For the given geometry and the dimensions used, the operation frequency of the sensor is $400 \mathrm{MHz}$ when the NSRR probe is loaded in free space. Considering that the maximum standard rebar grid period is around $10 \mathrm{~cm}$, it can be concluded that the rebar grid behaves as a perfect electric conductor at that wavelength. On the other hand, the size of the NSRR probe $(4.7 \mathrm{~cm} \times 4.7 \mathrm{~cm})$ is relatively small compared to the standard rebar grid period, and the NSRR probe only sees the effect of the part of the grid on which it is attached. Since the displacement or strain along one direction is measured, the NSRR probe is placed on the rebar such that the rebar is parallel to the tooth pairs. Therefore, the effect of the rebar grid on the sensor is the same as that of a single rebar electromagnetically. The cylindrical rebar introduces an additional capacitance in parallel to the effective NSRR capacitance, and thus the resonance frequency is decreased. As expected, this decrease is related to the diameter of the rebar; a larger diameter implies more additional capacitance and a further reduced frequency. In [34], it was shown that the sensor is able to measure displacement and strain successfully when attached on a rebar, which is pulled by a high-scale loading setup. However, in that case, the $8 \mathrm{~mm}$ diameter steel rebar touching the backside of the NSRR probe also results in a frequency drop of about $100 \mathrm{MHz}$. But as demonstrated in [34], this decrease does not hinder the operation of the sensor; only the numerical fit parameters used for the calibration should be changed.

In the first set of experiments in complex media, a rebar grid is placed behind the NSRR probe at a $1 \mathrm{~cm}$ distance. The antenna monitors the NSRR from its front side as in the previous measurements. Behind the NSRR probe, a 2-D fragment of the 3-dimensional rebar grid is placed (see Fig. 7), as normally would appear in a real-life scenario, only without concrete. This fragment of the rebar grid consists of three parallel $16 \mathrm{~mm}$ diameter rebars separated by a gap of $9 \mathrm{~cm}$ and four perpendicularly placed $10 \mathrm{~mm}$ diameter rebars separated by a gap of $10 \mathrm{~cm}$. The fragment of the rebar grid can be placed either in a position such that the thicker rebars are in vertical (Fig. 7-a and Fig. 7-c) or in horizontal position (Fig. 7-b and Fig. 7-d). The former and latter positions are denoted as vertical and horizontal placements, respectively. The difference between these two positions is that in the vertical position, the $10 \mathrm{~mm}$ diameter rebars lie along the direction of the comb-like NSRR tooth pairs, i.e., along the direction of displacement; while in the horizontal position, the $16 \mathrm{~mm}$ diameter rebars lie along the direction

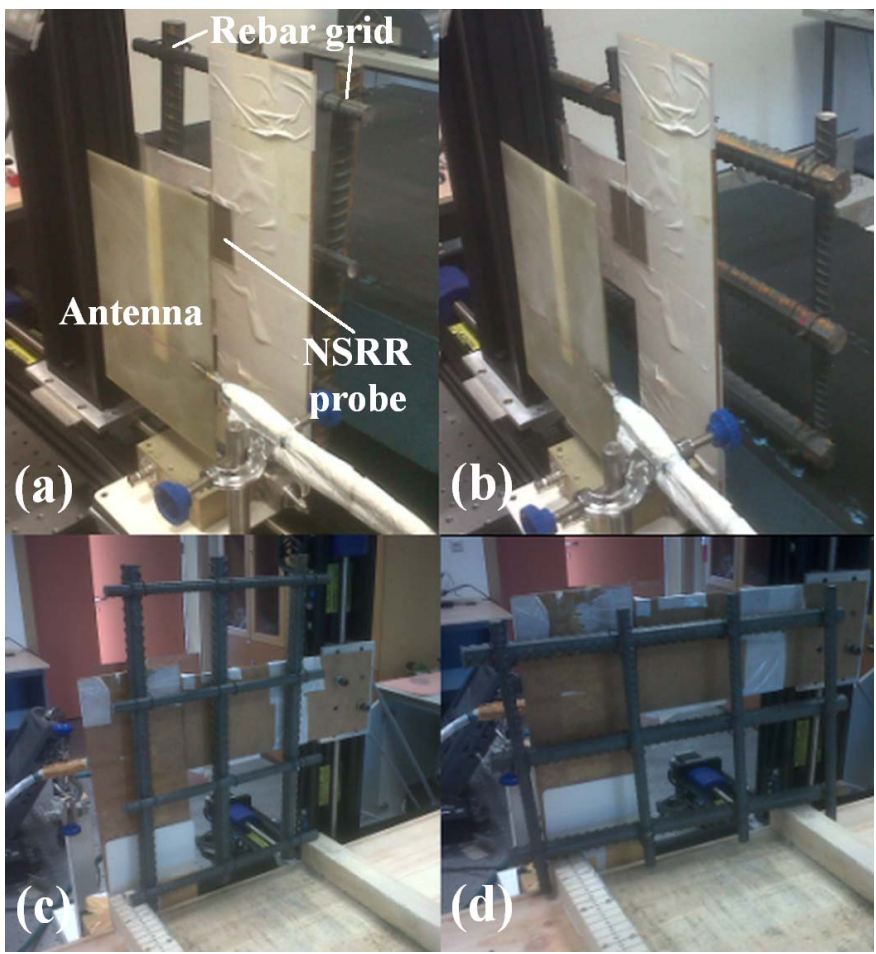

Fig. 7. A fragment of the rebar grid placed behind the NSRR probe in (a) vertical and (b) horizontal positions. The backside images are given in (c) and (d)

TABLE I

Modification of Fit Parameters When a Rebar Grid Is Placed BEHIND THE NSRR PROBE IN VERTICAL AND HORIZONTAL POSITIONS

\begin{tabular}{|c||c||c|}
\hline & $k_{1}(\mathrm{MHz} / \mathrm{mm})$ & $k_{2}(\mathrm{MHz})$ \\
\hline No rebar & 24.3 & 404 \\
\hline Vertical & 22.8 & 399 \\
\hline Horizontal & 21.5 & 303 \\
\hline
\end{tabular}

of displacement. As pointed out, the electromagnetic effects of these two positions are naturally different. The horizontal placement is expected to introduce more additional capacitance to the structure due to larger metal presence at the back of the NSRR, thus leading to a lower resonance frequency compared to both the no-rebar case and the vertical placement case. The frequency shift versus displacement is shown for the no-rebar case as well as for the vertical and horizontal placements of the rebar grid in Fig. 8. The jumper length is $2 \mathrm{~cm}$ for the measurements. The exponential fit explained in Section III is also applied to each case in Fig. 8. In each fit, different values for $k_{1}$ and $k_{2}$ are used, while other parameters remain unchanged. The set of the fit parameters used in each case are shown in Table I.

Fig. 8 shows that the placement of a rebar grid $1 \mathrm{~cm}$ away decreases the resonance frequency approximately by 10 and $20 \mathrm{MHz}$ in the vertical and horizontal positions, respectively. As mentioned before, the worst case occurs when the sensor is attached on the rebar in a touching position, leading to a decrease up to $100 \mathrm{MHz}$ for a $8 \mathrm{~mm}$ diameter rebar [34]. However, even in that case, the sensor operation is not hindered and frequency shift can be detected. 


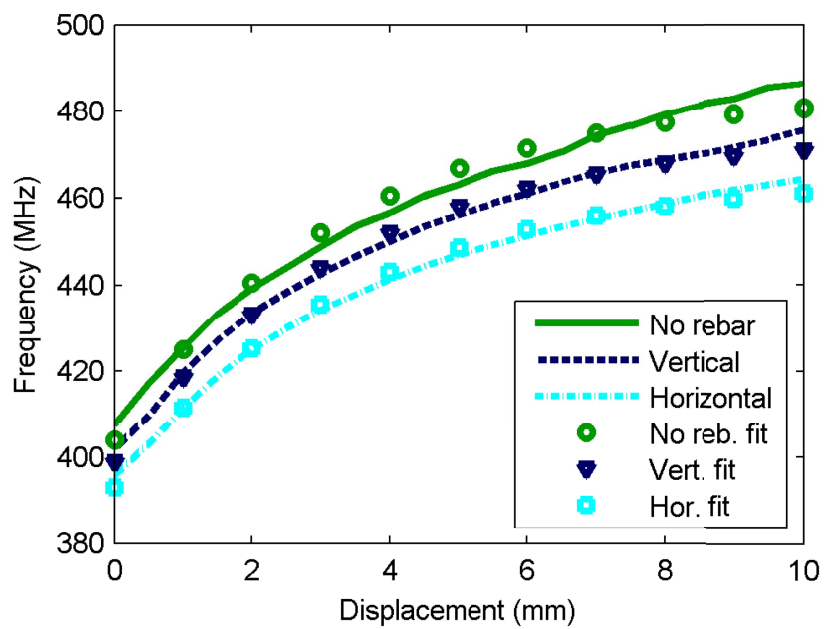

Fig. 8. Translational measurement of the change of sensor resonance frequency with $d$ in the no-rebar case and for the cases of vertical and horizontal placement of the rebar grid fragment behind the NSRR probe, shown along with the exponential fits for each case. $l=2 \mathrm{~cm}$ in all cases.

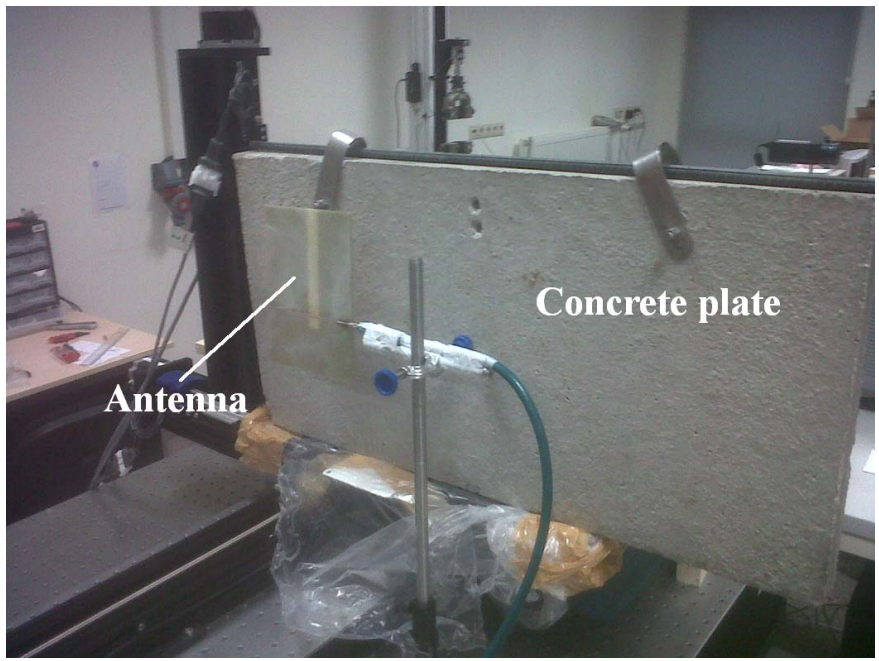

Fig. 9. Experimental setup of the scenario in which a $4 \mathrm{~cm}$ thick concrete plate is present between the antenna and the NSRR probe.

An experiment in which a relatively thin concrete plate is placed between the NSRR probe and the antenna to represent the concrete cover, is the next step to understand the sensor's performance towards real-life scenarios. For this purpose, a $4 \mathrm{~cm}$ thick concrete plate with surface dimensions of $70 \mathrm{~cm} \times 35 \mathrm{~cm}$ is used (see Fig. 9). Since it is known that concrete is not a perfectly homogeneous material, the translation stage experiment is repeated for several different positions of the plate to check the effect of each case on the sensor operation. These positions include translation in the horizontal position to left and right with respect to a predetermined origin (center), elevation in the upwards direction, and $180^{\circ}$ reverting of the concrete plate around its axis. Each scenario is expected to create minor changes on the variation of the sensor frequency with displacement. These changes are presented in Fig. 10, where $l=4 \mathrm{~cm}$ for all measurements. This set of experiments shows that the effect

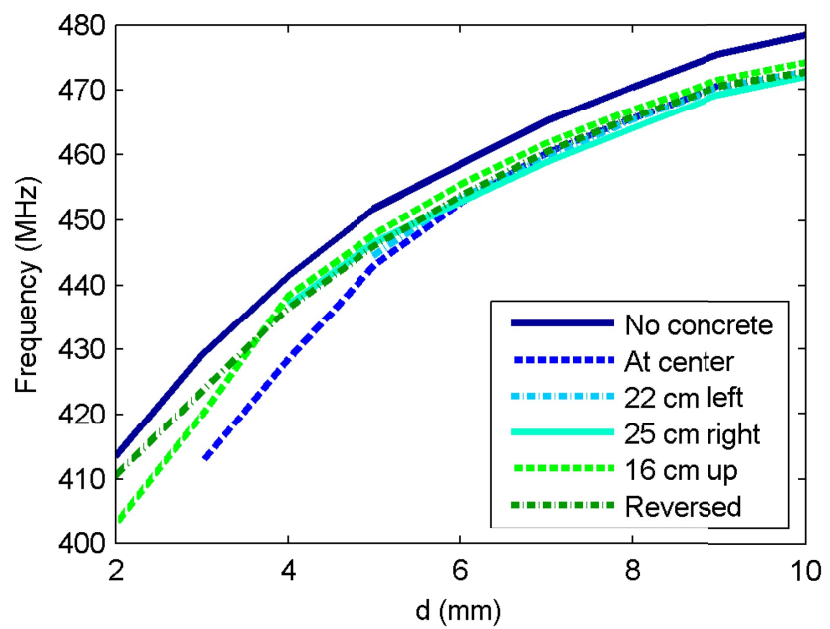

Fig. 10. Measured change of the sensor frequency with varying displacement for different placements of the $4 \mathrm{~cm}$ thick concrete plate between the antenna and the NSRR probe.

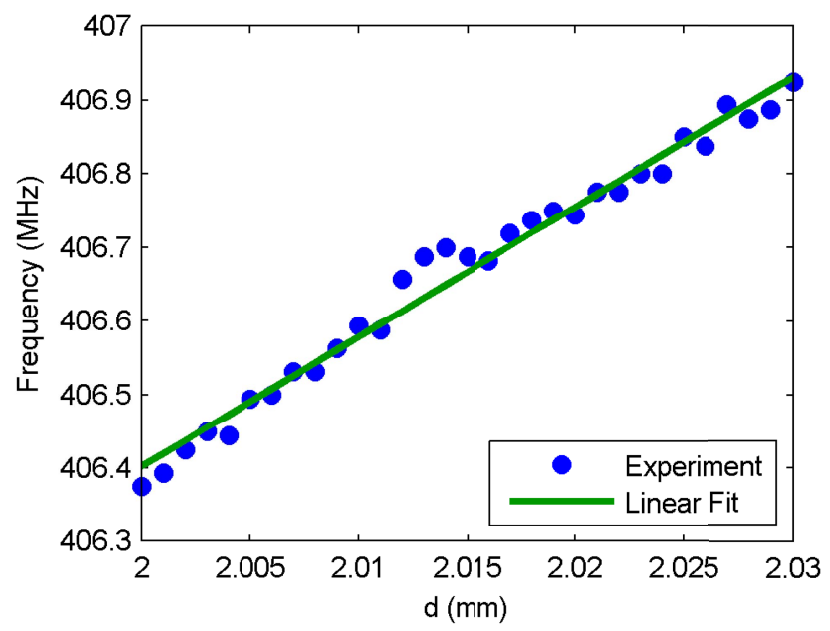

Fig. 11. Measured resolution of the sensor in the presence of a 4-cm-thick concrete plate: Sensor frequency versus $d$ for a $30 \mu \mathrm{m}$ range with $1 \mu \mathrm{m}$ steps, where $l=4 \mathrm{~cm}$.

of the concrete as a cover placed between the sensor elements is not very significant, creating a frequency decrease of at most $15 \mathrm{MHz}$ at any $d$ value. This also demonstrates that the concrete block can be considered as a homogeneous medium for our measurements, and the detection of frequency change behind the cover does not depend on the antenna position.

The resolution of the sensor was shown to be at the sub- $\mu \mathrm{m}$ level for measurements in free space, in [33]. The effect of the presence of a concrete cover between the antenna and the NSRR probe on the resolution is investigated as the next characterization. The experiment is performed by using a Newport DM-13 micrometer, which allows for a $0.5 \mu \mathrm{m}$ minimum graduation level. The step size is selected as $1 \mu \mathrm{m}$ for a $d$ range of $30 \mu \mathrm{m}$. $l$ is $4 \mathrm{~cm}$ for the NSRR probe in the experiment. The frequency change with $d$ is shown in Fig. 11. It is observed that the concrete cover slightly decreases the resolution, from sub- $\mu \mathrm{m}$ to $1 \mu \mathrm{m}$ level. Despite that, to the best of the authors' knowledge, 


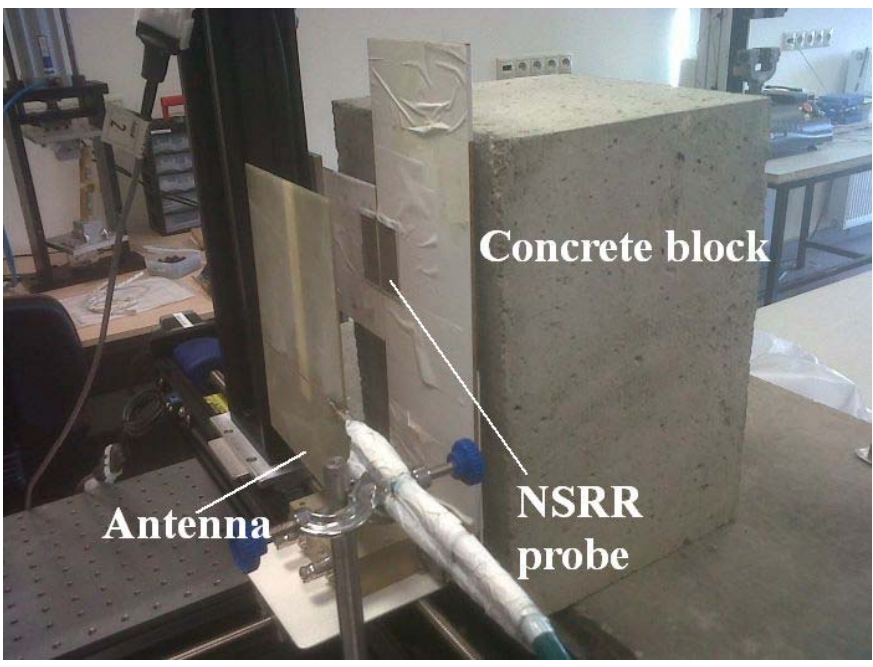

Fig. 12. Experimental setup for the measurement of frequency change versus displacement when a concrete block is present behind the NSRR probe.

this level of resolution in the complex medium is still better than those of the previously shown passive wireless sensors for SHM in literature, some of which were reported in free space [5], [18], [37]. The concrete plate experiments related with the drop of the resonance frequency and the resolution hold significance because the front cover is the only element whose effect on the sensor cannot be avoided. The problems introduced by complex medium elements like rebar and concrete core that stay behind the NSRR probe are easier to deal with; on the other hand, the cover always stands between the antenna and the sensing probe and its effect is inevitable. Since the performance of the sensor is not affected significantly by the concrete cover, the proposed scheme is highly favorable for structural health monitoring. The dynamic range is also shown to be large, which is basically limited by $l$, and can extend up to over $20 \mathrm{~mm}$ as shown in [33]. Sensitivity also remains slightly affected and is still excellent, with an average of around $7.5 \mathrm{MHz} / \mathrm{mm}$ in the worst case over the whole measured range.

The placement of a rebar grid behind the NSRR probe, or that of a concrete plate between the antenna and the sensor is shown here to affect the sensor performance at a minimal level. However, placing a concrete block behind the NSRR probe can be expected to change the sensor characteristics due to the lossy nature of concrete. To understand these effects, a concrete block with the dimensions $20 \mathrm{~cm} \times 20 \mathrm{~cm} \times 30 \mathrm{~cm}$ is brought closer to the NSRR probe from behind on the translation stage. The experimental setup and the measured change of resonance frequency with $d$ are shown in Fig. 12 and Fig. 13, respectively. The jumper length $l$ is $4 \mathrm{~cm}$ for all measurements. In Fig. 13, the numerical fits applied to the experimental data are also shown for each distance. These plots demonstrate that the presence of a concrete block behind the NSRR starts to disrupt the signal at a distance between $3-5 \mathrm{~cm}$, and for the distances below this value, the frequency continues to decrease further. The reason is again the additional capacitance introduced by the concrete block, and below a specific distance, it dominates the sensor response. In Fig. 13, it is observed that the total

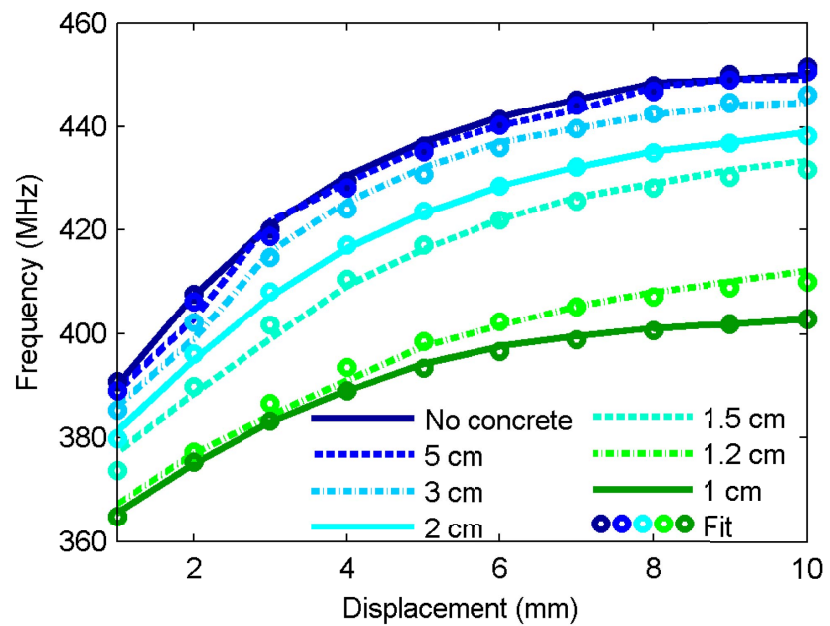

Fig. 13. Measured change of sensor frequency when the concrete block is behind the NSRR probe, shown for several concrete block distances. The exponential fits are also shown.

TABLE II

Modification of Fit Parameters When a Concrete Block Is Placed Behind the NSRR Probe at SeVERAl Distances

\begin{tabular}{|c||c||c|}
\hline & $k_{1}(\mathrm{MHz} / \mathrm{mm})$ & $k_{2}(\mathrm{MHz})$ \\
\hline No concrete block & 26.4 & 368 \\
\hline Concrete at $5 \mathrm{~cm}$ & 26.7 & 366 \\
\hline Concrete at $3 \mathrm{~cm}$ & 26.4 & 363 \\
\hline Concrete at $2 \mathrm{~cm}$ & 25.4 & 358 \\
\hline Concrete at $1.5 \mathrm{~cm}$ & 25.2 & 352 \\
\hline Concrete at $1.2 \mathrm{~cm}$ & 19.7 & 348 \\
\hline Concrete at $1 \mathrm{~cm}$ & 16.5 & 351 \\
\hline
\end{tabular}

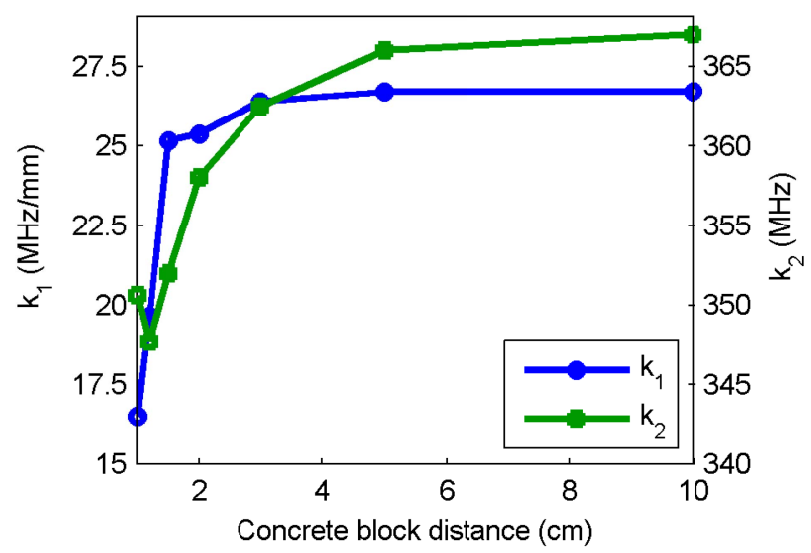

Fig. 14. Graphical representation of the change of the fit parameters $k_{1}$ and $k_{2}$ with concrete block distance to the NSRR probe.

frequency change, that is, the frequency difference between $d=10 \mathrm{~mm}$ and $d=0 \mathrm{~mm}$, also decreases as the concrete block is brought closer to the NSRR probe. This is different than the effect of changing $l$, which is shown to introduce only a frequency offset at all $d$ values in Fig. 4. This impact can be described in the best way by observing the change of fit parameters $k_{1}$ and $k_{2}$ again, while other parameters remain the same. Table II gives the fit parameters for every concrete block distance, and Fig. 14 shows the variation of these parameters graphically. We can understand that both the 
slope and the average of the frequency shift curves are subject to an exponential decrease as concrete block is brought closer, accelerating even further for distances below approximately $1.5 \mathrm{~cm}$. Since the sensitivity of the sensor is defined as the change of frequency over a range of $d$, the placement of the concrete block behind the NSRR probe causes a severe loss of sensitivity. For the distances shorter than $1 \mathrm{~cm}$, the concrete block starts to disrupt the coupling of the system even more gravely, basically to the point that the frequency shifts start to be overwhelmed with the capacitive loading of the concrete background. Therefore, it can be deduced that a minimum distance of $1 \mathrm{~cm}$ should be kept between the existing NSRR probe geometry and the reinforced or unreinforced concrete. With the effect of the inevitable concrete cover already shown to be minimal, a thorough sensor operation can be anticipated provided that this distance is preserved. The easiest way to maintain such a separation is to back the NSRR probe with an air-like dielectric material, that is, to fill the gap between the concrete core and the NSRR with a material whose electromagnetic properties are close to those of free space. A $1 \mathrm{~cm}$ thick separator fabricated from such a material can be split into two halves and can be fastened to the comb-like NSRR probe parts and to the rebar without difficulty. The idea is to use the separator in the same shape with the NSRR geometry and to have it freely moving with forming displacements so that the strain is directly transfered to the probe. One possible choice for the separator material is the polystyrene foam since it is cheap and commonly used and its dielectric constant of 2.5 is one of the closest to free space.

In a real life scenario, the rebar grid is generally embedded inside the concrete behind the NSRR probe and it may be expected to change the electromagnetic behavior of the complex medium. Yet another factor that can affect this behavior is the age of the concrete. It is known that the electromagnetic properties of the concrete are subject to change with time, as it dries slowly. In order to test these effects, four concrete blocks with the same dimensions as the one shown in Fig. 12 are prepared. The first block incorporates a 3-dimensional rebar grid. The second block, which is prepared one month later, does not contain any rebar grid inside. The third and fourth blocks are prepared at the same day and two months after the second block, and one of them contains a rebar grid while the other does not. In the experiment, these concrete samples are placed at a $2 \mathrm{~cm}$ distance behind the NSRR probe with a jumper length of $4 \mathrm{~cm}$. The comparison of the results for the last two blocks is useful in understanding the difference between a reinforced concrete sample and an unreinforced one which are prepared on the same day. On the other hand, comparison of the first and second cases with the last two cases gives an idea on the effect of the concrete age on the sensor performance. Therefore, the effects created due to both the age and the rebar grid content of the concrete blocks can be comprehended by these experiments. The change of frequency for all these samples are shown in Fig. 15.

Fig. 15 shows that even though there is a variation of frequency shifting regime among different combinations of reinforced and unreinforced concrete blocks with varying ages,

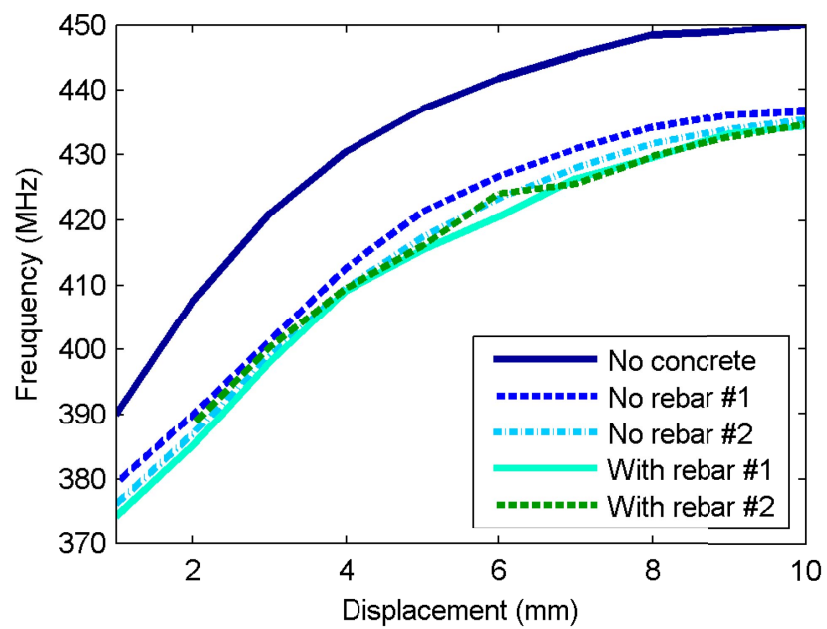

Fig. 15. Change of frequency with displacement for three different concrete samples with varying ages and rebar grid presence. The blocks are placed at a distance of $2 \mathrm{~cm}$ behind the NSRR probe.

this variation is only around $6 \mathrm{MHz}$ at maximum for any $d$ value. The deviation of this beam of curves from the no-concrete case is due to the disruptive nature of the concrete block itself. Hence, the age factor, and whether the concrete is reinforced or unreinforced makes a little change in terms of the sensor performance.

\section{CONCLUSION}

In this work, the performance of a near-field wireless displacement and strain sensor is investigated in the presence of an electromagnetically complex medium constituted by different combinations of the rebar grid and concrete. The results show that although these components all give rise to a changed resonance frequency of the coupled system to some degree, the sensor can still operate in real-life SHM conditions. The $4 \mathrm{~cm}$ thick concrete cover placed between the antenna and the NSRR probe is shown only to scarcely affect the sensor operation. The rebar grid content inside the concrete block and the age of the concrete sample are also shown to be of minor importance, and different combinations of these parameters yield similar frequency-displacement curves.

The most detrimental case is observed when a concrete block is present immediately behind the NSRR. To overcome the disruptive effects of the concrete core behind the NSRR, placement of a separator material with air-like electromagnetic properties between the probe and the concrete core is proposed. These effects are the most prominent when the distance between the NSRR and the concrete is less than $1 \mathrm{~cm}$, therefore the thickness of the separator can be selected to be $1 \mathrm{~cm}$. This, with the addition of the concrete cover, is a case which mimics the real-life SHM application the most closely. The fact that the sensor operates well in such a case, showing a resolution of $1 \mu \mathrm{m}$, is very promising. This resolution is the best in comparison to the similar wireless displacement sensors available in the literature. The high resolution and sensitivity are thought to result from the high near-field coupling between the antenna and the used comb-like NSRR probe geometry 
specially designed for SHM. The major drawback of such a system is the monitoring (interrogation) distance, which is limited to around $80 \mathrm{~cm}$. The proposed sensor has a high dynamic range of over $20 \mathrm{~mm}$, which is achieved when the measurable displacement is made independent of the elastic modulus of the NSRR probe material by splitting it into two halves.

For the extraction of displacement (or strain) information from the measured frequency, a numerical fit is also proposed in this work. The parameters of the fit are shown to depend on the complex medium, and this dependency is examined for several complex medium scenarios. Utilizing such a numerical fit, the translational measurements, therefore, serve as a calibration for the real-life applications. For a given complex medium geometry, the fit parameters can be determined beforehand on the translation stage, and the mapping between the measured frequency shift and displacement (or strain) can be extracted via these fit parameters. The fact that the sensor can operate in real life conditions with slightly degraded performance is promising in vitally important application areas including SHM.

\section{REFERENCES}

[1] E. L. Tan, A. J. DeRouin, and K. G. Ong, "Magnetoelastic-harmonic stress sensors with tunable sensitivity," IEEE Sensors J., vol. 12, no. 6, pp. 1878-1883, Jun. 2012.

[2] B. D. Pereles, A. J. DeRouin, T. A. Dienhart, E. L. Tan, and K. G. Ong, "A wireless, magnetoelastic-based sensor array for force monitoring on a hard surface," Sensor Lett., vol. 10, nos. 3-4, pp. 806-813, Mar. 2012.

[3] Y. Jia, K. Sun, F. J. Agosto, and M. T. Quiñones, "Design and characterization of a passive wireless strain sensor," Meas. Sci. Technol. vol. 17, no. 11, pp. 2869-2876, Sep. 2006

[4] S.-D. Jang, B.-W. Kang, and J. Kim, "Frequency selective surface based passive wireless sensor for structural health monitoring," Smart Mater. Struct., vol. 22, no. 2, p. 025002, 2013.

[5] D. J. Thomson, D. Card, and G. E. Bridges, "RF cavity passive wireless sensors with time-domain gating-based interrogation for SHM of civil structures," IEEE Sensors J., vol. 9, no. 11, pp. 1430-1438, Nov. 2009.

[6] M. J. Cazeca, J. Mead, J. Chen, and R. Nagarajan, "Passive wireless displacement sensor based on RFID technology," Sens. Actuators A, Phys., vol. 190, pp. 197-202, Feb. 2013.

[7] A. Vaz et al., "Full passive UHF tag with a temperature sensor suitable for human body temperature monitoring," IEEE Trans. Circuits Syst. II, Exp. Briefs, vol. 57, no. 2, pp. 95-99, Feb. 2010.

[8] M. K. Jain, Q. Cai, and C. A. Grimes, "A wireless micro-sensor for simultaneous measurement of $\mathrm{pH}$, temperature, and pressure," Smart Mater. Struct., vol. 10, no. 2, pp. 347-353, 2001.

[9] A. J. DeRouin, S. J. Trierweiler, B. D. Pereles, B. Lippi, and K. G. Ong, "A low cost, wireless embedded sensor for moisture monitoring in hardto-access places," Sensor Lett., vol. 11, no. 9, pp. 1573-1578, Sep. 2013.

[10] A. D. DeHennis and K. D. Wise, "A wireless microsystem for the remote sensing of pressure, temperature, and relative humidity," J. Microelectromech. Syst., vol. 14, no. 1, pp. 12-22, Feb. 2005.

[11] K. J. Loh, J. P. Lynch, and N. A. Kotov, "Inductively coupled nanocomposite wireless strain and pH sensors," Smart Struct. Syst., vol. 4, no. 5, pp. 531-548, Sep. 2008.

[12] B. E. Horton, S. Schweitzer, A. J. DeRouin, and K. G. Ong, "A varactorbased, inductively coupled wireless pH sensor," IEEE Sensors J., vol. 11, no. 4, pp. 1061-1066, Apr. 2011.

[13] Q. Y. Cai, A. Cammers-Goodwin, and C. A. Grimes, "A wireless, remote query magnetoelastic $\mathrm{CO}_{2}$ sensor," J. Environ. Monitor., vol. 2, no. 6, pp. 556-560, Dec. 2000.

[14] K. G. Ong, M. Paulose, and C. A. Grimes, "A wireless, passive, magnetically-soft harmonic sensor for monitoring sodium hypochlorite concentrations in water," Sensors, vol. 3, no. 1, pp. 11-14, Jan. 2003.
[15] M. M. Andringa, D. P. Neikirk, and S. L. Wood, "Unpowered wireless analog resistance sensor," Proc. SPIE Smart Struct. Mater., Sensors Smart Struct. Technol. Civil, Mech., Aerosp. Syst., vol. 5391, p. 356, Jul. 2004

[16] A. Mita and S. Takhira, "A smart sensor using a mechanical memory for structural health monitoring of a damage-controlled building," Smart Mater. Struct., vol. 12, no. 2, pp. 204-209, 2003.

[17] S. Deshmukh and H. Huang, "Wireless interrogation of passive antenna sensors," Meas. Sci. Technol., vol. 21, no. 3, p. 035201, Jan. 2010.

[18] C. Occhiuzzi, C. Paggi, and G. Marrocco, "Passive RFID strainsensor based on meander-line antennas," IEEE Trans. Antennas Propag., vol. 59, no. 12, pp. 4836-4840, Dec. 2011.

[19] A. Daliri, A. Galehdar, S. John, C. H. Wang, W. S. T. Rowe, and K. Ghorbani, "Wireless strain measurement using circular microstrip patch antennas," Sens. Actuators A, Phys., vol. 184, pp. 86-92, Sep. 2012.

[20] T. T. Thai, H. Aubert, P. Pons, G. DeJean, M. M. Tentzeris, and R. Plana, "Novel design of a highly sensitive RF strain transducer for passive and remote sensing in two dimensions," IEEE Trans. Microw. Theory Techn., vol. 61, no. 3, pp. 1385-1396, Mar. 2013.

[21] A. K. Horestani, C. Fumeaux, S. F. Al-Sarawi, and D. Abbott, "Displacement sensor based on diamond-shaped tapered split ring resonator," IEEE Sensors J., vol. 13, no. 4, pp. 1153-1160, Apr. 2013.

[22] M. Hasani, A. Vena, L. Sydänheimo, L. Ukkonen, and M. M. Tentzeris, "Implementation of a dual-interrogation-mode embroidered RFIDenabled strain sensor," IEEE Antennas Wireless Propag. Lett., vol. 12, no. 10, pp. 1272-1275, Oct. 2013

[23] A. Vena et al., "An embroidered two-dimensional chipless strain sensor for wireless structural deformation monitoring," IEEE Sensors J., vol. 13 no. 12 , pp. 4627-4637, Dec. 2013.

[24] C. Paggi, C. Occhiuzzi, and G. Marrocco, "Sub-millimeter displacement sensing by passive UHF RFID antennas," IEEE Trans. Antennas Propag., vol. 62, no. 2, pp. 905-912, Feb. 2014.

[25] A. K. Horestani, J. Naqui, Z. Shaterian, D. Abbott, C. Fumeaux, and F. Martín, "Two-dimensional alignment and displacement sensor based on movable broadside-coupled split ring resonators," Sens. Actuators A, Phys., vol. 210, pp. 18-24, Apr. 2014.

[26] W. Su, O. A. Hazim, I. L. Al-Qadi, and S. M. Riad, "Permittivity of Portland cement concrete at low RF frequencies," Mater. Eval., vol. 52 no. 4, pp. 496-502, Mar. 1994.

[27] H. C. Rhim and O. Buyukozturk, "Electromagnetic properties of concrete at microwave frequency range," ACI Mater. J., vol. 95, no. 3, pp. 262-271, May 1998.

[28] R. A. Dalke, C. L. Holloway, P. McKenna, M. Johansson, and A. S. Ali, "Effects of reinforced concrete structures on RF communications," IEEE Trans. Electromagn. Compat., vol. 42, no. 4, pp. 486-496, Nov. 2000.

[29] E. Richalot, M. Bonilla, M.-F. Wong, V. Fouad-Hanna, H. Baudrand, and J. Wiart, "Electromagnetic propagation into reinforced-concrete walls," IEEE Trans. Microw. Theory Techn., vol. 48, no. 3, pp. 357-366, Mar. 2000.

[30] R. Paknys, "Reflection and transmission by reinforced concreteNumerical and asymptotic analysis," IEEE Trans. Antennas Propag., vol. 51, no. 10, pp. 2852-2861, Oct. 2003.

[31] M. Dehmollaian and K. Sarabandi, "An approximate solution of scattering from reinforced concrete walls," IEEE Trans. Antennas Propag., vol. 56, no. 8, pp. 2681-2690, Aug. 2008.

[32] S. Jiang and S. V. Georgakopoulos, "Optimum wireless powering of sensors embedded in concrete," IEEE Trans. Antennas Propag., vol. 60, no. 2, pp. 1106-1113, Feb. 2012.

[33] B. Ozbey et al., "Wireless displacement sensing enabled by metamaterial probes for remote structural health monitoring," Sensors, vol. 14, no. 1, pp. 1691-1704, Jan. 2014.

[34] B. Ozbey, H. V. Demir, O. Kurc, V. B. Erturk, and A. Altintas, "Wireless measurement of elastic and plastic deformation by a metamaterial-based sensor," Sensors, vol. 14, no. 10, pp. 19609-19621, Oct. 2014.

[35] R. Melik et al., "Nested metamaterials for wireless strain sensing," IEEE J. Sel. Topics Quantum Electron., vol. 16, no. 2, pp. 450-458, Mar./Apr. 2010.

[36] S. I. Latif, L. Shafai, and S. K. Sharma, "Bandwidth enhancement and size reduction of microstrip slot antennas," IEEE Trans. Antennas Propag., vol. 53, no. 3, pp. 994-1003, Mar. 2005.

[37] I. Mohammad and H. Huang, "Monitoring fatigue crack growth and opening using antenna sensors," Smart Mater. Struct., vol. 19, no. 5, p. $055023,2010$. 


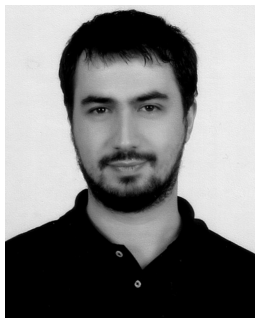

Burak Ozbey (S'12) was born in Ankara, Turkey, in 1986. He received the B.S. and M.S. degrees in electrical and electronics engineering from Bilkent University, Ankara, Turkey, in 2008 and 2011, respectively, where he is currently pursuing the $\mathrm{Ph} . \mathrm{D}$. degree. His research interests include electromagnetic theory, microwave circuits, metamaterials, and design and testing of wireless RF sensors.

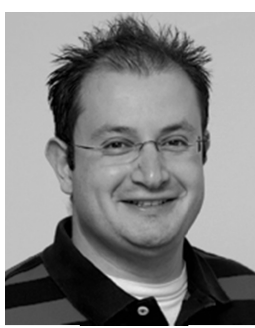

Hilmi Volkan Demir received the M.S. and $\mathrm{Ph} . \mathrm{D}$. degrees from Stanford University. He is currently a Professor of Electrical Engineering and Physics. He is the EURYI Associate Professor with Bilkent University, Turkey. Concurrently, he is named a Fellow by the Singapore National Research Foundation and appointed to a Nanyang Associate Professorship at Nanyang Technological University, Singapore. He serves as the Director of the Luminous! Center of Excellence. Among his research interests are semiconductor device physics and wireless sensing. He has contributed to commercialization and licensing of several new enabling technologies and establishing a successful company and led to more than 30 patent applications, several of which have currently been used, owned or licensed by the industry. These scientific and entrepreneurship activities resulted in important international awards, including the Nanyang Award for Research Excellence, the European Science Foundation European Young Investigator Award, and The Outstanding Young Person in the World Award from the JCI Federation of Young Leaders and Entrepreneurs.

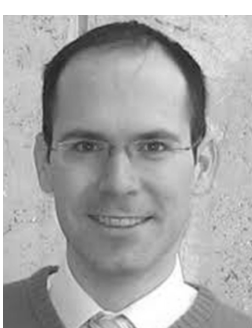

buildings.

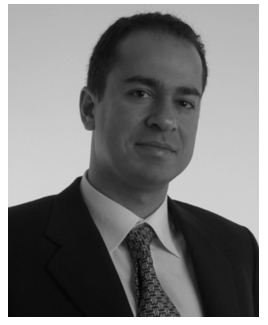

Vakur B. Ertürk (M'00) received the B.S. degree in electrical engineering from Middle East Technical University, Ankara, Turkey, in 1993, and the M.S. and Ph.D. degrees from The Ohio-State University, Columbus, in 1996 and 2000, respectively. He is currently an Associate Professor with the Electrical and Electronics Engineering Department, Bilkent University, Ankara. His research interests include the analysis and design of planar and conformal arrays, high-frequency techniques, structural health monitoring, magnetic resonance imaging, scattering from and propagation over large terrain profiles. He served as the Secretary/Treasurer of the IEEE Turkey Section and the Turkey Chapter of the IEEE Antennas and Propagation, Microwave Theory and Techniques, Electron Devices, and Electromagnetic Compatibility Societies. He was a recipient of the 2005 URSI Young Scientist and the 2007 Turkish Academy of Sciences Distinguished Young Scientist Awards.

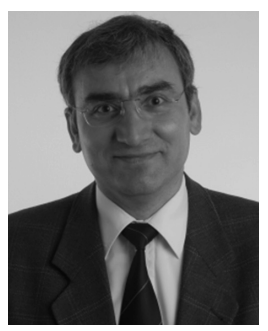

Ayhan Altintas (SM'93) received the B.S. and M.S. degrees from Middle East Technical University (METU), Ankara, Turkey, in 1979 and 1981, respectively, and the Ph.D. degree from The Ohio State University, Columbus, in 1986. From 1981 to 1987, he was with the ElectroScience Laboratory, The Ohio State University. He is currently a Professor of Electrical Engineering with Bilkent University, Ankara, Turkey. He has held research fellow and guest professor positions at Australian National University, Canberra, Australia, the Tokyo Institute of Technology, Japan, the Technical University of Munich, Germany, and Concordia University, Montreal, Canada. His research interests include high frequency and numerical techniques in electromagnetic scattering and diffraction, propagation modeling and simulation, and fiber and integrated optics. He is a member of Sigma Xi and Phi Kappa Phi. He is a Fulbright Scholar, and an Alexander von Humboldt Fellow. He is the President of the URSI Turkish National Committee. He was a recipient of the IEEE Third Millennium Medal. He received the ElectroScience Laboratory Outstanding Dissertation Award of 1986, the IEEE 1991 Outstanding Student Branch Counselor Award, the 1991 Research Award of Prof. Mustafa N. Parlar Foundation of METU, and the Young Scientist Award of Scientific and Technical Research Council of Turkey, Tubitak, in 1996. He was the Chairman of the IEEE Turkey Section for the terms 1991-1993 and 1995-1997. 